\title{
Comparison of Diffusion and Transport in human head
}

\author{
Anna Custo and David A. Boas
}

What: Diffuse Optical Imaging (DOI) is a relatively new method used to image blood volume and oxygen saturation in vivo. It uses near infrared light and has the advantage of low cost and portability. We compare two well-known forward models for photon migration in the human head: Monte Carlo (MC) of the transport equation [6] and Finite-Difference of the diffusion equation (FD) [1].

Why: Due to the long processing time associated with Monte Carlo, it is advisable to adopt a faster alternative forward model with comparable accuracy. FD, implementing the diffusion equation, offers greater computational efficiency, but at the cost of modeling accuracy. The low scattering properties of the Cerebral Spinal Fluid (CSF) filling the space between the brain and the skull has been of particular concern in the development of an accurate photon migration forward problem for the human head as the diffusion equation is known to provide inaccurate solutions under such circumstances $[1,4]$. The roughness of CSF is of particular interest as it limits the average straight-line distance that a photon would travel in the "void" region. Thus, even if the "void" region does not scatter light, we could treat it as if it had an effective scattering coefficient such that the typical scattering length is greater than the average straight-line distance through the "void" region [1]. The diffusion equation may be perfectly accurate under such conditions.

How: The head model we employ is provided by MRI segmented data. With such adult head geometry we can specify up to five tissue types (scalp, skull, CSF, gray and white matter) but for most of our test we use three (as described in Table 1). The whole volume is voxelized in a cube with 256 voxel each side (2563 voxel in total, $1 \mathrm{~mm}^{3}$ each) or 1283 voxels, $2 \mathrm{~mm}^{3}$ each; two different resolution is used in order to enhance each forward model performance. The interesting tissue types are immerged into air (tissue type 0 ). The optical properties are lined out in Table 1.

\begin{tabular}{|l|c|c|}
\hline Tissue Types & Absorption coefficient $\left[\mathrm{mm}^{-1}\right]$ & Transport scattering coefficient $\left[\mathrm{mm}^{-1}\right]$ \\
\hline Scalp and Skull & 0.019 & 0.86 \\
\hline CSF & 0.004 & $0.001,0.01,0.1,0.2,0.3,0.7,1.0$ \\
\hline Brain & 0.01 & 1.11 \\
\hline
\end{tabular}

The single source and the 25 detectors are placed on the top-left corner of the head and the detectors placement follows a linear scheme (all detectors are placed on the same plane as the source). The Monte Carlo (MC) method models individual photon trajectories through the various tissues, reproducing the casualty of each scatter event in a stochastic fashion (random seed is employed). When the photon is detected, its residual weight, reduced during its traveling through the tissues at each scatter event, is calculated from $\mu_{a}$ and partial optical path length for each tissue type passed through. MC has disadvantage of requiring high computational time (being computationally expensive) to produce data with a significant Signal-to-Noise Ratio (SNR). Finite-Difference (FD) code provides a solution to the diffusion equation. However, relies on assumptions that break down at early times and for very small scattering coefficients. Boundary Conditions (BC) are fundamental to the model accuracy. The run time of this code is extremely short (on the order of minutes instead of several hours like the MC solver).

Progress: We run several tests (such as Partial Optical Path length Factor (PPF) in time domain and continuous wave, Temporal Point Spread Function, Spatial Sensitivity Profile) using Monte Carlo simulation in order to investigate the importance of a good characterization of CSF reduced scattering coefficient. The data collected prove that the presence of CSF is important in an accurate head model but its scattering coefficient will not greatly affect Monte Carlo predictions if varying between 0.3 and 0.001 $\mathrm{mm}^{-1}$ (for a CSF layer not thicker than $4 \mathrm{~mm}$ ).

The comparison of PPF predicted by FD and MC for CW measurements (Fig. 1) and TD for a head model with CSF $\mu_{s} 1.0$ (model 1) and $0.1 \mathrm{~mm}^{-1}$ (model 2) leads to results similar to the reported on previous works $[4,2,5,3]$, but the discrepancy between FD and MC is smaller than previously stated. Error bars shown in Fig. 1 display the standard error calculated combining 11 independent MC run, each one simulating one hundred million photons. Observing the qualitative response of MC and FD in CW 

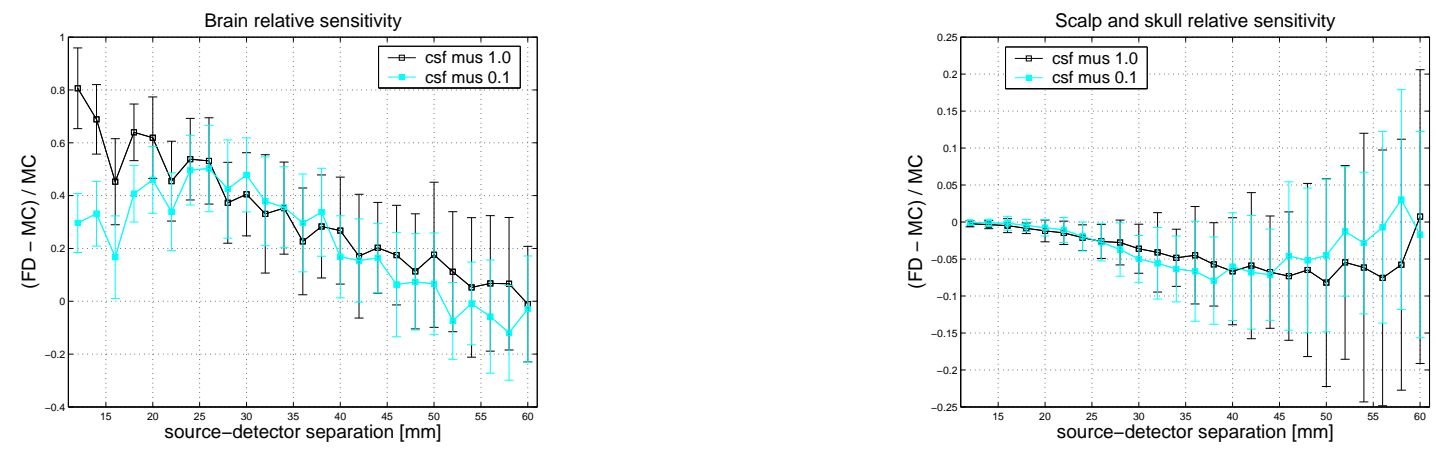

Figure 1: Relative Partial Optical Path length Factor (PPF) for scalp-skull layer (left) and brain (right) employing a head model with CSF scattering coefficient 1.0 (empty squares) and $0.1 \mathrm{~mm}^{-1}$ (full squares). Standard Error is shown along with the relative sensitivity to absorption changes

and TD we conclude that Diffusion based methods can well predict photon scattering through biological tissues in a complex 3D geometry. We observe 37.5\% difference at early time (and small distance from the source), that becomes as small as $13.3 \%$ in detected signal within $35 \mathrm{~mm}$ from the source.

Future: Remains to be explored the effect that the measured discrepancy of the two forward models has on the inverse problem, which is, when restoring the head optical properties. In functional study of brain activity, calculating the liner forward model to employ in the inverse process is typically computationally expensive. The use of FD method as suppose to MC simulation saves computational time and allows a better and faster tuning of the regularization parameters involved in the linear restoration process. Various inverse models can be explored and methods combining diverse imaging sources (such as DOI, MRI and fMRI) can be tested for an optimal reconstruction of the head optical parameters.

\section{References:}

[1] A.H. Barnett, J.P. Culver, A.G. Sorensen, A. Dale, and D.A. Boas. Robust inference of baseline optical properties of the human head with $3 \mathrm{~d}$ segmentation from magnetic resonance imaging. Applied Optics, 42 (16):3095 - 3108, 2003.

[2] Y. Fukui, Y. Ajichi, and E. Okada. Monte carlo prediction of near-infrared light propagation in realistic adult and neonatal head models. Applied Optics, page 42 (16), 2003.

[3] T. Hayashi, Y. Kashio, and E. Okada. Hybrid monte carlo-diffusion method for light propagation in tissue with a low-scattering region. Applied Optics, page 42 (16), 2003.

[4] E. Okada, M. Firbank, and D.T. Delpy. The effect of overlying tissue on the spatial sensitivity profile of near-infrared spectroscopy. Phys. Med. Biol., page 40, 1995.

[5] E. Okada, M. Firbank, M. Schweiger, S.R. Arridge, M. Cope, and D.T. Delpy. Theoretical and experimental investigation of the near-infrared light propagation in a model of the adult head. Applied Optics, page 36 (1), 1997.

[6] L. Wang, S.L. Jacques, and L. Zheng. Mcml - monte carlo modeling of light transport in multi-layered tissues. Computer Methods and Programs in Biomedicine, pages 47, 131-146, 1995. 